Khan, M. S. J.; Wang, Y.-W.; Senge, M. O.; Peng, Y. (2018):

Sensitive fluorescence on-off probes for the fast detection of a chemical warfare agent mimic. Journal of Hazardous Materials 342, 10-19. doi: 10.1016/j.jhazmat.2017.08.009

\title{
Sensitive fluorescence on-off probes for the fast detection of a chemical warfare agent mimic
}

\author{
Muhammad Shar Jhahan Khan, ${ }^{\mathrm{a}}$ Ya-Wen Wang, ${ }^{\mathrm{a}}{ }^{*}$ Mathias O. Senge, ${ }^{\mathrm{b}}$ and Yu Peng ${ }^{\mathrm{a}, *}$ \\ ${ }^{a}$ State Key Laboratory of Applied Organic Chemistry, Lanzhou University, Lanzhou, Gansu \\ 730000, Peoples Republic of China \\ ${ }^{b}$ Trinity Translational Medicine Institute, Medicinal Chemistry, Trinity Centre for Health \\ Sciences, St. James's Hospital, Trinity College Dublin, The University of Dublin, Dublin 8, \\ Ireland \\ * Corresponding author. \\ E-mail addresses: ywwang@1zu.edu.cn, pengyu@1zu.edu.cn.
}

\begin{abstract}
Two highly sensitive probes bearing a nucleophilic imine moiety have been utilized for the selective detection of chemical warfare agent (CWA) mimics. Diethyl chlorophosphate (DCP) was used as mimic CWAs. Both iminocoumarin-benzothiazole-based probes not only demonstrated a remarkable fluorescence ON-OFF response and good recognition, but also exhibited fast response times (10 seconds) along with color changes upon addition of DCP. Limits of detection for the two sensors $\mathbf{1}$ and $\mathbf{2}$ were calculated as 0.06 $\mu \mathrm{M}$ and $0.21 \mu \mathrm{M}$, respectively, which are much lower than most other reported probes. These two probes not only show high sensitivity and selectivity in solution, but can also be applied for the recognition of DCP in the gas state, with significant color changes easily observed by the naked eye.
\end{abstract}

Keywords: fluorescence probe, chemical warfare agents, diethyl cholorophosphate, recognition

\section{Introduction}

Organophosphorus compounds (OPs) are essential compounds for use in agriculture as pesticides and herbicides. However, large scale use presents a significant health hazard and their high toxicity facilitated military uses as chemical warfare agents (CWAs). Notably, nerve agents of the non-persistent G- (Tabun, Sarin, Soman, Cyclosarin) and persistent Vseries (VE, VG, VM, VX) are prominent OP CWAs and are classified by the United Nations as weapons of mass destruction [1,2]. These agents cause a blockage of the 
acetylcholinesterase enzyme in Human, resulting in disruption of nerve impulse transmission and death [3]. While the military use of such OPs CWAs is highly restricted by international laws, recurring use in conflict zones and by terrorists makes them a contemporary and persistent threat $[4,5]$. Thus, detection of such nerve agents by easy and convenient analytical methods is critically important both for the military under battlefield conditions, for security forces tasked with preventing their manufacture and use, and for the public health system to assure early detection [6].

Over the years a range of methods has been developed for the detection of nerve agents and these range from mass spectrometry, photoacoustics, biosensing, electrical sensors, capillary electrophoresis, etc. [2,7-12]. However, the use of such instrumental or enzymatic methods is often expensive, complex, and not sensitive enough and does not lend itself to use under field conditions. Thus, alternative approaches target OP degradation products and/or use immunological or spectroscopic methods $[13,14]$ or feature a supramolecular chemistry strategy [15].

Likewise, many of these problems can be overcome using optical sensors and notably fluorescence probes are emerging as suitable alternatives due to their ease of handling, low cost and high sensitivity [16-21].

While interest in highly selective and sensitive fluorescence probes for the detection of chemical warfare agents is increasing, the number of chemically distinct classes of such sensors is still limited [22-27]. Some probes allow naked eye detection facilitated by color modulations, a more convenient feature for conditions and situations where fluorimeters are not available. General characteristics for suitable probes are highly selectivity, fast response times, low limits of detection and high sensitivity in response to the specific chemicals $[28,29]$. Additionally, facile synthesis or use of existing sensors, a simple analytical reaction and ease of handling would be beneficial from a commercial perspective and we have reported on such systems for the detection of anions and cations, among others [30,31]

For OP-type nerve agents one such approach entails the use of a simple chemical reaction of a nucleophilic probe with the electrophilic P-center of OPs to generate a product spectroscopically distinct from the probe alone. Here, iminocoumarins [32] are attractive targets, as reactions at the imino group are accompanied by significant spectroscopic changes suitable for the detection of anions and biothiols as reported by us [33,34].

In practical terms research on CWA detection does not use the original nerve gases due to high toxicity and regulatory issues but utilizes mimics or simulants of the nerve agents such 
as diethyl chlorophosphate (DCP) which exhibit similar chemical behavior but much lower toxicity (Fig. 1) [15]. With the development of fluorescence probes in our group [30,31,3339], herein we report the use of two iminocoumarin-benzothiazole-based probes $(\mathbf{1}, \mathbf{2})$ to recognize and report DCP as mimic for G-series nerve agents through significant fluorescence and color changes.

\section{Experimental Section}

\subsection{Materials}

Commercial compounds such as diethyl chlorophosphate (DCP) from Acros Organics, dimethyl methylphosphonate (DMMP) from Adamas-beta, or synthetic starting materials (benzothiazole-2-acetonitrile from Damas-beta, 8-hydroxyjulolidine-9-carboxaldehyde from J\&K Chemical, 4-(diethylamino)salicylaldehyde from J\&K Chemical) were of analytical grade and used as received without any purification. To deal with the problem of hydrolysis of $\mathrm{DCP}$ and $\mathrm{HCl}$ generation in the reaction mixture, further experiments were carried out in HEPES buffer and in the presence of high concentration of $\mathrm{HCl}$. Probes $\mathbf{1}$ and 2 were prepared according to published procedures and gave analytical data in agreement to the literature [32]. Details of the synthesis of 1, 2, 3 and $\mathbf{4}$ and characterization of all compounds are given in SI in Fig. S1-S12.

\subsection{Methods}

Fluorescence spectroscopy studies were carried out with a Hitachi F-7000 fluorescence spectrophotometer. The wavelength range was $420-780 \mathrm{~nm}$ with a scan rate of $1200 \mathrm{~nm} \mathrm{~min}{ }^{-1}$. Solutions were measured in $1.0 \mathrm{~cm}$ path length quartz cell. Water used for HEPES buffer was triply distilled. The excitation and emission slit width was $2.5 \mathrm{~nm}$. Absorption spectra were measured with a Cary 5000 UV-Vis-NIR spectrophotometer where solutions were measured in $1.0 \mathrm{~cm}$ path length quartz cell. Baseline correction measurements were carried out for all spectra. Solvents used were of analytical grade and all the fluorescence and UV-Vis experiments were carried out at room temperature. NMR spectra were obtained with a Bruker ADVANCE III $400 \mathrm{MHz}$ instrument using TMS as an internal standard. ESI-MS were recorded on ESQ6K esquire6000 instrument. Melting points were determined on a Kofler apparatus. All single-crustal X-ray was performed on a Bruker smart CCD area detector 
diffractometer. Fluorescence quantum yields were determined in solution, using fluorescein $(\Phi=0.85$ in $0.1 \mathrm{M} \mathrm{NaOH})$ as a standard.

\section{Results and discussion}

\subsection{Probe design and sensing mechanism}

The probes suitable for the detection of OPs as CWA mimics should ideally give a rapid and sensitive spectroscopic response, preferably accompanied by a naked-eye observable color change. Such chromo-reactant approaches have been used before for the identification of CWA mimics using xenthenes based on photo-induced electron transfer process (PET), enzyme based sensors, cyclodextrins, supermolecular hydrogel based probes etc. $[21,22,40,41]$. Based on our previous experience with using iminocoumarins for to $\mathrm{Hg}^{2+}$, $\mathrm{Cu}^{2+}$, fluorides and for bioimaging applications, we chose the two benzothiazole derivatives 1 and 2 as an alternative. They are easily prepared in a one-step reaction from commercial precursors [32] and have shown excellent response for DCP based on their fluorescence. Furthermore, the presence of the imino functionality should present a simple synthetic handle for reaction with the electrophilic center of OP-based CWA mimics and thus native CWAs. The irreversibility of such a reaction with the analyte should provide a convenient sensing mechanism and unambiguous product formation. With iminocoumarin-benzothiazoles this concept has thus far only been applied to sensing of thiophenol with 1 [42]; additionally its reaction with $\mathrm{N}$-nucleophiles has been studied [43].

Indeed, a test reaction of probe $\mathbf{1}$ with DCP and triethylamine as base gave the adduct $\mathbf{3}$ in $44 \%$ yield (Fig. 2). The reaction mechanism involves a standard nucleophilic attack of the imine lone electron pair at the phosphorus atom, and loss of the $\mathrm{Cl}$ leaving group as $\mathrm{HCl}$. A similar reaction of $\mathbf{3}$ with DCP gave adduct 4 in $39 \%$ yield. Formation of the two products is clearly indicated in the respective ${ }^{1} \mathrm{H}$ NMR spectra (Fig. 3).

For example, Fig. 3a shows the ${ }^{1} \mathrm{H}$ NMR spectrum of probe $\mathbf{1}$ and Fig. $3 \mathrm{~d}$ shows that of probe 2, with the signal for the oximate resonance appearing at $8.61 \mathrm{ppm}$; an indication for the presence of an active $\mathrm{NH}$ hydrogen atom in the probe $\mathbf{1}$ while for probe $\mathbf{2}$, it is at 8.80 ppm. This signal is absent in the spectra of the DCP adducts 3 and $\mathbf{4}$ (see Fig. 3c and 3f, respectively). Instead, new signals are observed at $8.92 \mathrm{ppm}$ for compound 3 and at $8.7 \mathrm{ppm}$ for compound 4. Moreover, the appearance of new resonance peaks at $1.42 \mathrm{ppm}$ in probe 3 and at $1.429 \mathrm{ppm}$ for $\mathbf{4}$ are representative for the ethoxy groups. The all signals of probe and 
adduct can be observed in Fig. $3 b$ and 3e, which are the spectra of $\mathbf{1}+$ DCP and $\mathbf{2}+$ DCP, respectively. Further evidence for the formation of the two adducts was derived from mass spectrometric studies, where the anticipated parent positive ion peaks at $m / z 486.6[\mathrm{M}+\mathrm{H}]^{+}$ and at $m / z 510.1840[\mathrm{M}+\mathrm{H}]^{+}$were observed for 3 and 4 , respectively and from X-ray crystallographic studies (see below).

\subsection{UV-Visible and Fluorescence Response}

Initial studies on possible sensing of DCP utilized UV-visible spectroscopy and we recorded the optical absorption spectra of probe $1(10.0 \mu \mathrm{M})$ and probe $2(10.0 \mu \mathrm{M})$ in the presence of diethyl chlorophosphate (DCP, $10.0 \mu \mathrm{M}$ ) to mimic the presence of a G-series nerve agent. For probe 1, the maximum absorption band appeared at $451 \mathrm{~nm}$ which was shifted bathochromically to $487 \mathrm{~nm}$ within less than $10 \mathrm{sec}$ with a color change from green to light gold (Fig. 4a). In the case of probe 2, a similar behavior was observed. The main absorption band at $481 \mathrm{~nm}$ was red-shifted to $513 \mathrm{~nm}$ upon addition of DCP, accompanied by a change in color from light green to pale pink (see Fig. 4b). The results clearly indicate that recognition of DCP can be realized using UV-visible spectroscopy. Notably, the short time response observed here is not very common [7,17,19, 44]. Moreover, absorption spectra of 3 $(10.0 \mu \mathrm{M})$ and $4(10.0 \mu \mathrm{M})$ were obtained, where $\lambda_{\mathrm{abs}}=487 \mathrm{~nm}$ for 3 and $\lambda_{\mathrm{abs}}=513 \mathrm{~nm}$ for 4 (Fig. S13). These results show that the UV-visible spectrum of compounds $\mathbf{3}$ or $\mathbf{4}$ was the same as the products of probe $\mathbf{1}$ or $\mathbf{2}$ with DCP.

Next, to determine the use of the iminocoumarins as fluorescence probes for chemical warfare agents fluorescence spectra were measured in different solvents such as acetonitrile, DMF and dichloromethane (see SI, Fig. S14 and S15). These investigations showed that probe 1 gave a better response to DCP in DMF, while probe 2 showed superior results in dichloromethane due to the solvent effect [45]. Hence, DMF was used as solvent for further investigation with $\mathbf{1}$ and dichloromethane for further studies with probe $\mathbf{2}$.

The fluorescence spectra of probe 1 and probe 2 are shown in Fig. 5a and 5b, respectively. Probe 1 exhibited maximum emission at $526 \mathrm{~nm}$ and probe 2 at $531 \mathrm{~nm}$. After the addition of DCP the fluorescence intensity of both probes was quenched remarkably, accompanied by bathochromic shifts to $542 \mathrm{~nm}$ for probe 1 and $554 \mathrm{~nm}$ for probe 2, respectively. Again, this response was observed within a very short time frame of less than 10 seconds. This remarkable quenching $[4,5,46,47]$ shows the strong affinity between the probes and DCP. 
The fluorescence quantum yield $\left(\Phi_{F}\right)$ decreased from 0.15 to 0.03 (for probe 1) and from 0.79 to 0.27 (for probe 2) after addition of DCP. In addition to the strong fluorescence response, color modulations also took place in both probes. The fluorescence color of probe $\mathbf{1}$ changed from green to light yellow while for probe 2 a change from green to pink yellow was observed. These changes are easily observed by naked eye, as shown in the insets of Fig. 5 .

Titration studies were carried out to further investigate the quenching phenomenon. In case of probe 1 the fluorescence emission band at $526 \mathrm{~nm}$ (at $531 \mathrm{~nm}$ in the case of probe 2) gradually decreased with increasing amounts of DCP. Probe 1 showed a loss in fluorescence intensity of up to $91 \%$ while probe 2 was quenched up to $89 \%$ (see Fig. 5c and 5d). These results suggested the high sensitivity of the probes for DCP. The titration studies also allowed a determination of the limit of detection for probe 1 and $\mathbf{2}$ with DCP, which were found to be $0.06 \mu \mathrm{M}$ and $0.21 \mu \mathrm{M}$, respectively (SI, Fig. 16 and S17). These LOD values are lower than many of the reported work in this area [48]. As an example, A. K. Das et al. showed a LOD of $3.09 \mu \mathrm{M}$ for DCNP (a nerve agent mimic). Moreover, the fluorescence response of compounds 3 and 4 was also measured in the presence of the respective probes (SI, Fig. S18). This confirmed that the products of probe $\mathbf{1}$ and $\mathbf{2}$ with DCP are non-fluorescent and the fluorescence of the probes is not affected by the presence of the analyte adducts.

In order to study the selectivity of the probes, analogous experiments were performed with probe 1 and $\mathbf{2}$ in the presence of other OPs, such as dimethyl methylphosphonate (DMMP), phosphoric acid, triethyl phosphate and diethyl chlorothiophosphate. A look at the fluorescence responses of the two probes after addition of several OPs clearly revealed the selectivity of the compounds. Both probes showed high selectivity for DCP as shown in Fig. 6. Only upon addition of DCP was the fluorescence intensity of probes $\mathbf{1}$ and $\mathbf{2}$ quenched significantly. Treatment of the probes with other OPs in solution only induced negligible fluorescence changes, but also gave no significant color changes as shown in Fig. 7.

\subsection{Application relevant tests}

CWA testing under ambient and field conditions requires probes with sufficient stability, reactivity and tolerance of a wide range of $\mathrm{pH}$ and solvent conditions. Thus, the $\mathrm{pH}$ profile for both probes in the presence and absence of DCP was determined (Fig.8). These results revealed that both the probes are efficient over a wide range of $\mathrm{pH}$, as shown in Fig 8. Probe 1 functions well in the $\mathrm{pH}$ range from 6.0 to 10.0 , while probe 2 is efficient at almost every 
$\mathrm{pH}$ value. However, maximum quenching was observed in both cases at $\mathrm{pH}$ 8.0. Therefore, both probes are suitable for field detection of nerve agents, i.e. in real chemical warfare situations under in moist and aerobic conditions.

Other aspects one has to consider are the use of the CWA mimics, which, upon time, may release $\mathrm{HCl}$ and their putative liability under acidic conditions. So to prove the legitimacy of reactions of probes 1 and 2 with DCP, further UV and fluorescence investigations of 1, 2, 3 and 4 were carried out in HEPES buffer and in the presence of high concentration of $\mathrm{HCl}$, as shown in SI Fig. S19-S25. From these studies it was confirmed that there was no significant influence of HEPES buffer and $\mathrm{HCl}$ in UV and fluorescence responses and that the probes are stable and useful under any condition.

Finally, to investigate the action of DCP vapors/fumes on probe $\mathbf{1}$ and $\mathbf{2}$, two flasks were utilized in such a manner that fumes of DCP could be trapped inside the flasks. One flask contained probe $1(10.0 \mu \mathrm{M})$ in DMF and the second flask contained probe $2(10.0 \mu \mathrm{M})$ in dichloromethane. Two small bottles containing DCP were placed in each flask, avoiding any contact of liquid DCP with the probes and both flasks were sealed from air and kept at room temperature. As soon as the DCP fumes came into contact with respective the probes, the color of both test solutions changes. As shown in Figure 9 within 30 minutes the color of probe 1 changed from green to light gold (Fig. 9a and 9b), while the color of probe 2 changed from green to pink yellow (Fig. 9c and 9d). This is a clear indication of the utility of the probes for use in practical sensing applications [22,28,41,49].

\subsection{Crystallographic and computational studies}

Crystals suitable for single crystal X-ray crystallographic studies were obtained for probe $\mathbf{1}$ and the two DCP adducts $\mathbf{3}$ and $\mathbf{4}$ by crystallization from the evaporation of dichloromethane (Fig. 10, Table S1 and S2). The structure of 1 shows that benzene ring and benzothiazole ring are almost coplanar and their dihedral angel is about $7.27^{\circ}$. Crystal structure determinations of $\mathbf{3}$ and $\mathbf{4}$ confirmed the basic structure of the molecules and the expected formation of N-P bonds in the probe-DCP adducts. The N-P bond lengths are $1.632 \AA$ and $1.647 \AA$ for compounds 3 and 4, respectively. Furthermore, the iminocoumarin and benzothiazole rings are essentially coplanar. The corresponding dihedral angles are $1.53^{\circ}$ and $3.96^{\circ}$ for compound $\mathbf{3}$ and $\mathbf{4}$, respectively. 
In order to study the intrinsic relationship of the molecular structure of probes $\mathbf{1}$ and $\mathbf{2}$ with the respective fluorescence response, computational studies were performed using the Gaussian 09 program (Fig. 11, S26-S29). In the optimized structures of probes 1 and 2, and the DCP adducts $\mathbf{3}$ and $\mathbf{4}$, the iminocoumarin and benzothiazole skeletons are essentially coplanar, with dihedral angles of $0.21^{\circ}, 2.40^{\circ}, 2.64^{\circ}$ and $1.42^{\circ}$, respectively (see SI, Fig. S26S29). Furthermore, these results are in good agreement with the corresponding crystallographic data (vide supra). Notably, the main contributing transition of $\mathbf{4}$ derives from HOMO to LUMO and HOMO to LUMO +1, and the ones of 1, 2, and $\mathbf{3}$ arise from HOMO to LUMO. As illustrated in the molecular orbitals plots, most of the electron density of probes 1 and $\mathbf{2}$ is spread over the whole molecule, which indicates that probes $\mathbf{1}$ and $\mathbf{2}$ should have strong fluorescence. On the other hand, the electron densities in the HOMO and LUMO of compound 3 and HOMO and LUMO or LUMO +1 of $\mathbf{4}$ is distributed on the iminocoumarin-benzothiazole skeletons and phosphate groups, respectively. This illustrates the internal charge transfer process which is responsible for the changes in the fluorescence spectra upon quenching.

\section{Conclusions}

In summary, two probes containing imino groups were reacted with DCP as a G-series nerve agent simulant. Formation of the products was accompanied by significant spectroscopic changes which could be used for the fluorescent detection of DCP. Not only did addition of DCP to the probes results in fluorescence quenching (on-off response) with excellent response times and sensitivity, but the concomitant color changes could be observed by naked eye. Other OPs failed to give any significant response, and thus, the two probes are highly sensitive and selective for nerve gas detection, including in the vapor state.

\section{Acknowledgements}

This work was supported by the National Natural Science Foundation of China (Nos. 21572091 and 21472075), Program for Changjiang Scholars and Innovative Research Team in University (IRT-15R28), and Science Foundation Ireland (SFI IvP 13/IA/1894).

\section{Supplementary Material}


Experimental synthetic procedures for 1, 2, 3 and 4, Solvent effect, LOD, UV and fluorescence response in HEPES buffer and $\mathrm{HCl}$, crystallography and optimized structures.

\section{References}

[1] F. Worek, J. Jenner, H. Thiermann, eds., Chemical Warfare Toxicology: Volume 1: Fundamental Aspects, Royal Society of Chemistry, Cambridge, 2016.

[2] K. Kim, O. G. Tsay, D. A. Atwood, D. G. Churchill, Destruction and Detection of Chemical Warfare Agents, Chem. Rev. 111 (2011) 5345-5403.

[3] J. Bajgar, Organophosphates/nerve agent poisoning: Mechanism of action, diagnosis, prophylaxis, and treatment, Adv. Clin. Chem. 38 (2004) 151-216.

[4] A. Kumar, Threats Emanating From Weapons of Mass Destruction, Journal of Chemical and Biological Weapons, 3 (2010) 5-8.

[5] K. Ganesan, S. K. Raza, R. Vijayaraghavan, Chemical warfare agents, J. Pharm. Bioallied Sci. 2 (2010) 166-178.

[6] R. J. Brennan, J. F. Waeckerle, T. W. Sharp, S. R. Lillibridge, Chemical warfare agents: Emergency medical and emergency public health issues, Ann. Emerg. Med. 34 (1999) 191204.

[7] C. E. Kientz, Chromatography and mass spectrometry of chemical warfare agents, toxins and related compounds: state of the art and future prospects, J. Chromatogr. A 814 (1998) 123.

[8] H. Sohn, S. Letant, M. J. Sailor, W. C. Trogler, Detection of fluorophosphonate chemical warfare agents by catalytic hydrolysis with a porous silicon interferometer, J. Am. Chem. Soc. 122 (2000) 5399-5400.

[9] J. P Novak, E. S. Snow, E. J. Houser, D. Park, J. L. Stepnowski, R. A. McGill, Nerve agent detection using networks of single-walled carbon nanotubes, Appl. Phys. Lett. 83 (2003) 4026-4028.

[10] D. Noort, H. P. Benschop, R. M. Black, Biomonitoring of exposure to chemical warfare agents: A review, Toxicol. Appl. Pharmacol. 184 (2002) 116-126.

[11] H. O. Michel, E. C. Gordon, J. Epstein, Detection and Estimation of Isopropyl methylphosphonofluoridate and O-ethyl S-disisopropylamineethylmethylphosphonothioate in Sea Water in Parts-per-Trillion level, Environ. Sci. Technol. 7 (1973) 1045-1049. 
[12] W. E. Steiner, S. J. Klopsch, W. A. English, B. H. Clowers, H. H. Hill, Detection of a chemical warfare agent simulant in various aerosol matrixes by ion mobility time-of-flight mass spectrometry, Anal. Chem. 77 (2005) 4792-4799.

[13] J. A. Ashley, C.-H. Lin, P. Wirsching, K. D. Janda, Monitoring Chemical Warfare Agents: A New Method for the Detection of Methylphosphonic Acid, Angew. Chem. Int. Ed. 38 (1999) 1793-1795.

[14] L. Jenkins, O. M. Uy, G. M. Murray, Polymer-based lanthanide luminescent sensor for detection of the hydrolysis product of the nerve agent soman in water, Anal. Chem. 71 (1999) 373-378.

[15] M. R. Sambrook, S. Notman, Supramolecular chemistry and chemical warfare agents: from fundamentals of recognition to catalysis and sensing, Chem. Soc. Rev. 42 (2013) 92519267.

[16] H.-J. Kim, J.-H. Lee, H. Lee, J.-H. Lee, J.-H. Lee, J.-H. Jung, J.-S. Kim, A mesoporous, silica-immobilized-nanoparticle colorimetric chemosensor for the detection of nerve agents, Adv. Funct. Mater. 21 (2011) 4035-4040.

[17] B. Diaz de Greňu, D. Moreno, T. Torroba, A. Berg, J. Gunnars, T. Nilsson, R. Nyman, M. J. Persson, I. Pettersson, P. Wästerby, Fluorescent discrimination between traces of chemical warfare agents and their mimics, J. Am. Chem. Soc. 136 (2014) 4125-4128.

[18] T. J. Dale, J. Rebek Jr., Fluorescent Sensors for Organophosphorus Nerve Agent Mimics, J. Am. Chem. Soc. 128 (2006) 4500-4501.

[19] H. Lee, H.-J. Kim, Novel fluorescent probe for the selective detection of organophosphorous nerve agents through a cascade reaction from oxime to nitrile via isoxazole, Tetrahedron 70 (2014) 2966-2970.

[20] W. Xuan, Y. Cao, J. Zhou, W. Wang, A FRET-based ratiometric fluorescent and colorimetric probe for the facile detection of organophosphonate nerve agent mimic DCP, Chem. Commun. 49 (2013) 10474-10476.

[21] A. B. Bon, A. M. Costero, S. Gil, F. Sancenon, R. M. Manez, Chromo-fluorogenic BODIPY-complexes for selective detection of V-type nerve agent surrogates, Chem.Commun. 50 (2014) 13289-13291.

[22] S. Royo, R. Martinez-Manez, F. Sancenon, A. M. Costero, M. Parra, S. Gil, Chromogenic and fluorogenic reagents for chemical warfare nerve agents' detection Chem.Commun. (2007) 4839-4847. 
[23] M. Burnworth, S. J. Rowan, C. Weder, Fluorescence sensors for the detection of chemical warfare agents, Chem. Eur. J. 13 (2007) 7828-7836.

[24] S.-W. Zhang, T. M. Swager, Fluorescent detection of chemical warfare agents: functional group specific ratiometric chemosensors, J. Am. Chem. Soc. 125 (2003) 34203421.

[25] Z. Lei, Y. Yang, A concise colorimetric and fluorimetric probe for sarin related threats designed via the "covalent-assembly" approach, J. Am. Chem. Soc. 136 (2014) 6594-6597 [26] J. M. Rathfon, Z. M. Al-Badri, R. Shunmugam, S. M. Berry, S. Pabba, R. S. Keynton, R. W. Cohn, G. N. Tew, Fluorimetric nerve gas sensing based on pyrene imines incorporated into films and sub-micrometer fibers, Adv. Funct. Mater. 19 (2009) 689-695.

[27] D. Knapton, M. Burnworth, C. Weder, S. J. Rowan, Fluorescent organometallic sensors for the detection of chemical-warfare-agent mimics, Angew. Chem. Int. Ed. 45 (2006) 58255829.

[28] M. R. Sambrook, J. R. Hiscock, A. Cook, A. C. Green, I. Holden, J. C. Vincent, P. A. Gale, Hydrogen bond-mediated recognition of the chemical warfare agent soman (GD), Chem. Commun. 48 (2012) 5605-5607.

[29] J. Russell, J. A. Berberich, G. E. Drevon, R. R. Koepsel, Biomaterials for mediation of chemical and biological warfare agents, Annu. Rev. Biomed. Eng. 5 (2003) 1-27.

[30] M. Dong, Y.-W. Wang, Y. Peng, highly selective ratiometric fluorescent sensing for $\mathrm{Hg}^{2+}$ and $\mathrm{Au}^{3+}$, respectively, in aqueous media, Org. Lett. 12 (2010) 5310-5313.

[31] M. Dong, Y. Peng, Y.-M. Dong, N. Tang, Y.-W. Wang, A selective, colorimetric and fluorescent chemodosimeter for relay recognition of fluoride and cyanide anions based on 1,1'-Binaphthyl scaffold, Org. Lett. 14 (2012) 130-133.

[32] K. Komatsu, Y. Urano, H. Kojima, T. Nagano, Development of an iminocoumarin-based zinc sensor for suitable ratiometric fluorescence imaging of neuronal zinc, J. Am. Chem. Soc. 129 (2007) 13447-13454.

[33] Y. Peng, Y.-M. Dong, M. Dong, Y.-W. Wang, A selective, sensitive, colorimetric and fluorescence probe for relay recognition of Fluoride and $\mathrm{Cu}$ (III) ions with "off-on-off" switching in ethanol-water solution, J. Org. Chem. 77 (2012) 9072-9080.

[34] Y.-L. Yang, F.-M. Zhang, Y.-W. Wang, B.-X. Zhang, R. Fang, J.-G. Fang, Y. Peng, An iminocoumarine sulphonamide based turn-on fluorescent probe for the detection of biothiols in aqueous solution, Chem. Asian J. 10 (2015) 422-426. 
[35] Y. Peng, A.-J. Zhang, M. Dong, Y.-W. Wang, A colorimetric and fluorescent chemosensor for detection of an explosive 2,4,6-trinitophenol (TNP), Chem. Commun. 47 (2011) 4505-4507.

[36] Y.-L. Yang, Y.-W. Wang, Y. Peng, A bifunctional, colorimetric and fluorescent probe for recognition of $\mathrm{Cu}^{2+}$ and $\mathrm{Hg}^{2+}$ and its application in molecular logic gate, Sci. China Chem. 57 (2014) 289-295.

[37] Y.-W. Wang, S.-B. Liu, Y.-L. Yang, P.-Z. Wang, A.-J. Zhang, Y. Peng, A Terbium(III)complex-based on-off fluorescent chemosensor for phosphate anions in aqueous solution and its application in logic gate, ACS Appl. Mater. Interfaces, 7 (2015) 4415-4422.

[38] Y.-W. Wang, S.-B. Liu, W.-J. Ling, Y. Peng, A fluorescent probe for relay recognition of homosysteine and group IIIA ions including Ga(III), Chem. Commun. 52 (2016) 827-830.

[39] Z.-H. Fu, X. Han, Y. Shao, J. Fang, Z.-H. Zhang, Y.-W. Wang, Y. Peng, Fluoresceinbased chromogenic and ratiometric fluorescence probe for highly selective detection of cysteine and its application in bioimaging, Anal. Chem. 89 (2017) 1937-1944.

[40] S. Royo, A. Costero, M. Parra, S. Gil, R. M. Manez, F. Sancenon, Chromogenic, specific detection of the nerve-agent mimic DCNP (a tabun mimic), Chem. Eur. J. 17 (2011) 69316934.

[41] R. Gotor, S. Royo, A. M. Costero, M. Parra, S. Gil, R. M. Manez, F. Sancenon, Nerve agent simulant detection by using chromogenic triaryl methane cation probes, Tetrahedron, 68 (2012) 8612-8616.

[42] D. Kand, P. S. Mandal, A. Datar, P Talukdar, Iminocoumarin based fluorophores: Indispensable scaffolds for rapid, selective and sensitive detection of thiophenol, Dyes Pigm. 106 (2014) 25-31.

[43] N. Y. Gorobets, A. V. Borisov, A. V. Silin, V. M. Nikitchenko, S. N. Kovalenko, Reactions of 3-(4-Aryl-2-thiazolyl)- and 3-(2-Benzothiazolyl)-2-iminocoumarins with NNucleophiles, Chem. Heterocycl. Comp. 38 (2002) 1389-1396.

[44] B. Bon, A. M. Costero, S. Gil, R. M. Manez, F. Sancenon, Selective chromo-fluorogenic detection of DFP (a sarin and soman mimic) and DCNP (a tabun mimic) with a unique probe based on a boron dipyrromethane (BODIPY) dye, Org. Bio. Chem. 12 (2014) 8745-8751 
[45] X. Liu, J. M. Cole, K. S. Low, Solvent Effects on the UV-vis Absorption and Emission of Optoelectronic Coumarins: a Comparison of Three Empirical Solvatochromic Models, J. Am. Chem. Soc. 28 (2013) 14731-14741.

[46] S. W. zhang, T. M. Swager, Fluorescent Detection of Chemical Warfare Agents: Functional Group Specific Ratiometric Chemosensors, J. Am. Chem. Soc. 125 (2003) 34203421.

[47] H. Zhang, Y. Xie, P. Wang, G. Chen, R. Liu, Y. -W. Lam, Y. Hu, Q. Zhu, H. Sun, An iminocoumarin benzothiazole-based fluorescent probe for imaging hydrogen sulfide in living cells, Talanta, 135 (2015) 149-154.

[48] A. K. Das, S. Goswami, C. K. Quah, H. -K. Fun, Relay recognition of $\mathrm{F}^{-}$and a nerveagent mimic diethyl cyano-phosphonate in mixed aqueous media: discrimination of diethyl cyanophosphonate and diethyl chlorophosphate by cyclization induced fluorescence enhancement, RSC advances, 6 (2016) 18711-18717.

[49] R. Zhu, J. M. Azzarelli, T. M. Swager, Wireless hazard badges to detect nerve-agent simulants, Angew. Chem. Int. Ed. 55 (2016) 9662-9666. 

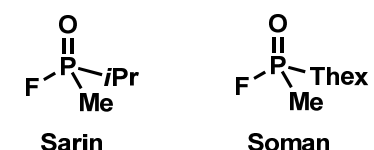

$\mathrm{NC}^{-}-\overbrace{\mathrm{OEt}}^{\mathrm{O}-\mathrm{NMM}_{2}}$

G-type nerve agents

Sarin

Soman

Tabun

O
DCP
II OEt

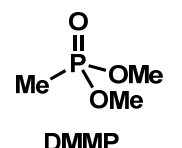

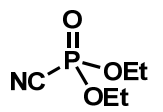

Mimics DCNP

Fig. 1. Chemical Warfare Agents and their Mimics. 

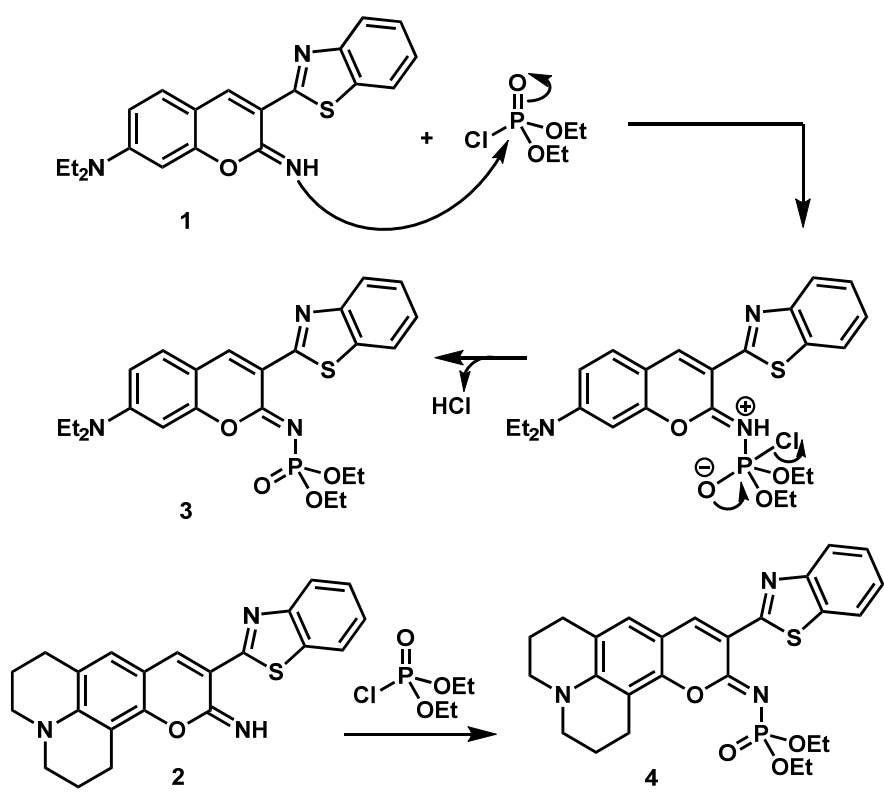

Fig. 2. Mechanism for the reaction of DCP with compound $\mathbf{1}$ to yield 3, and analogous reaction for the formation of 4. 

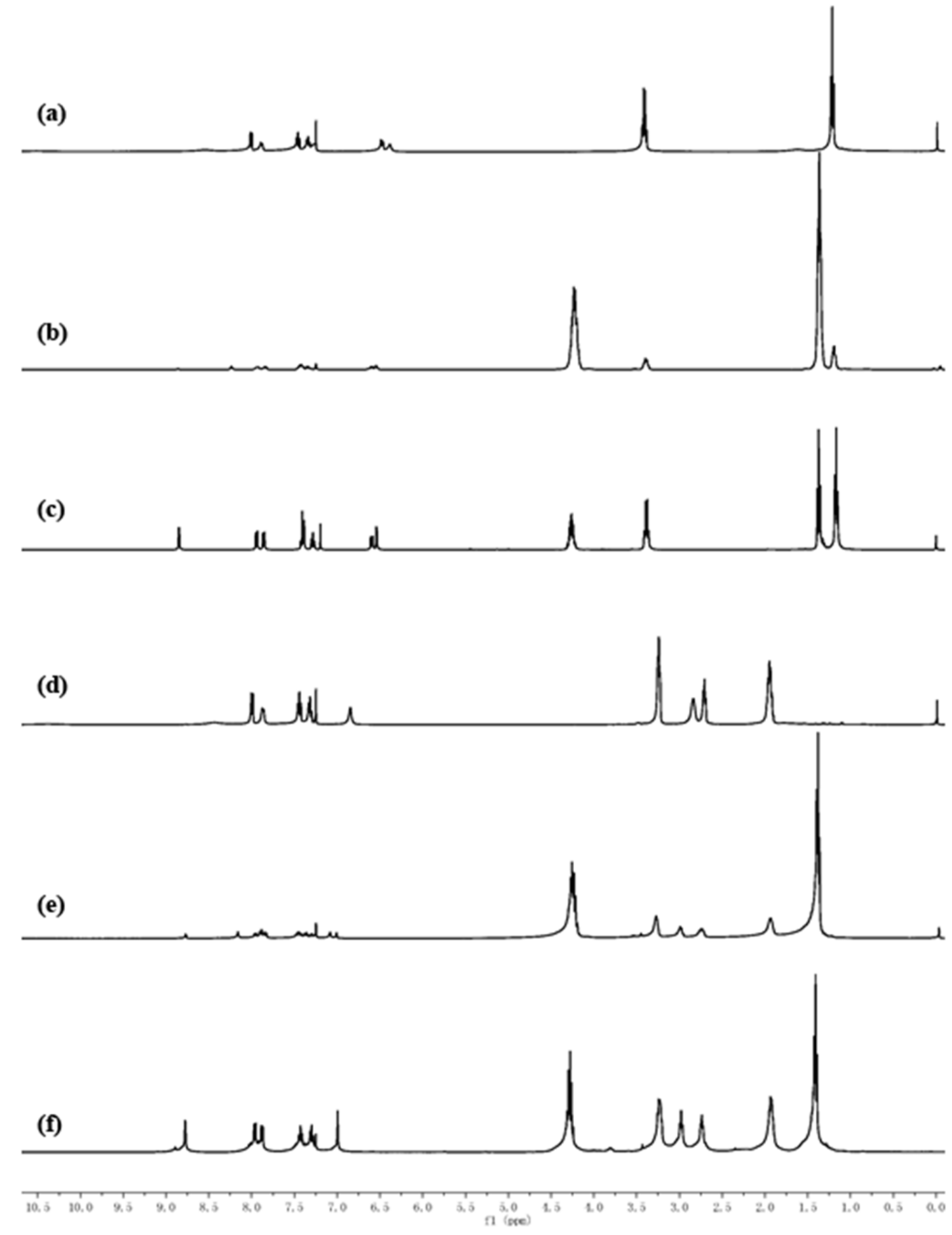

Fig. 3. ${ }^{1} \mathrm{H}$ NMR spectra of (a) probe 1, (b) probe $\mathbf{1}+\mathrm{DCP}$, (c) compound $\mathbf{3}$, (d) probe $\mathbf{2}$, (e) probe $\mathbf{2}+\mathrm{DCP}$, and (f) compound 4 in $\mathrm{CDCl}_{3}$. 

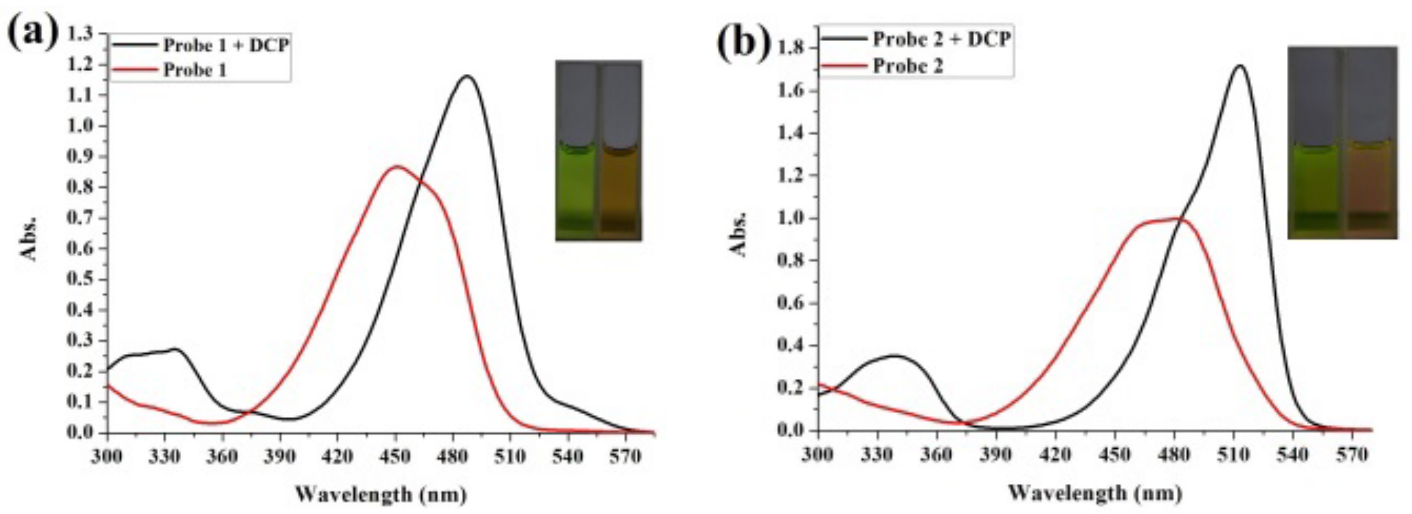

Fig. 4. UV spectra of (a) probe $1(10.0 \mu \mathrm{M})$ in DMF and after addition of DCP $(10.0 \mu \mathrm{M})$ and (b) probe 2 in DCM $(10.0 \mu \mathrm{M})$ and after addition of DCP $(10.0 \mu \mathrm{M})$ in DCM. Insets show the images of probe $\mathbf{1}$ and $\mathbf{2}$ free and after addition of DCP under visible light, respectively. 

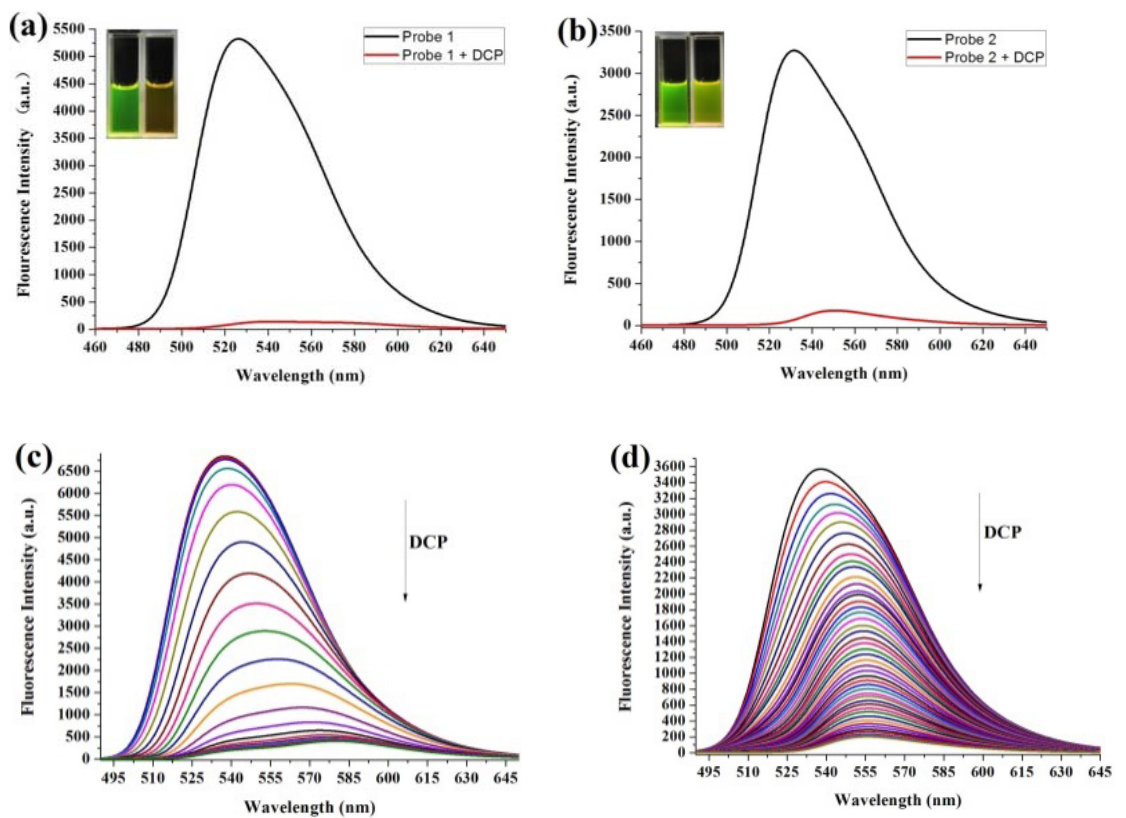

Fig. 5. Fluorescence spectra of (a) probe $1(10.0 \mu \mathrm{M})$ in DMF and after addition of DCP (10.0 $\mu \mathrm{M})$ and (b) probe $2(10.0 \mu \mathrm{M})$ in DCM and after addition of DCP $(10.0 \mu \mathrm{M})$; (c) titration of probe $1(25.0 \mu \mathrm{M})$ and DCP $(0.10 \mu \mathrm{M})$ in dichloromethane and (d) titration of probe $2(25.0 \mu \mathrm{M})$ with DCP $(0.10 \mu \mathrm{M})$ in dichloromethane. Insets show images of probe $\mathbf{1}$ and $\mathbf{2}$ free and after addition of DCP under UV light. 

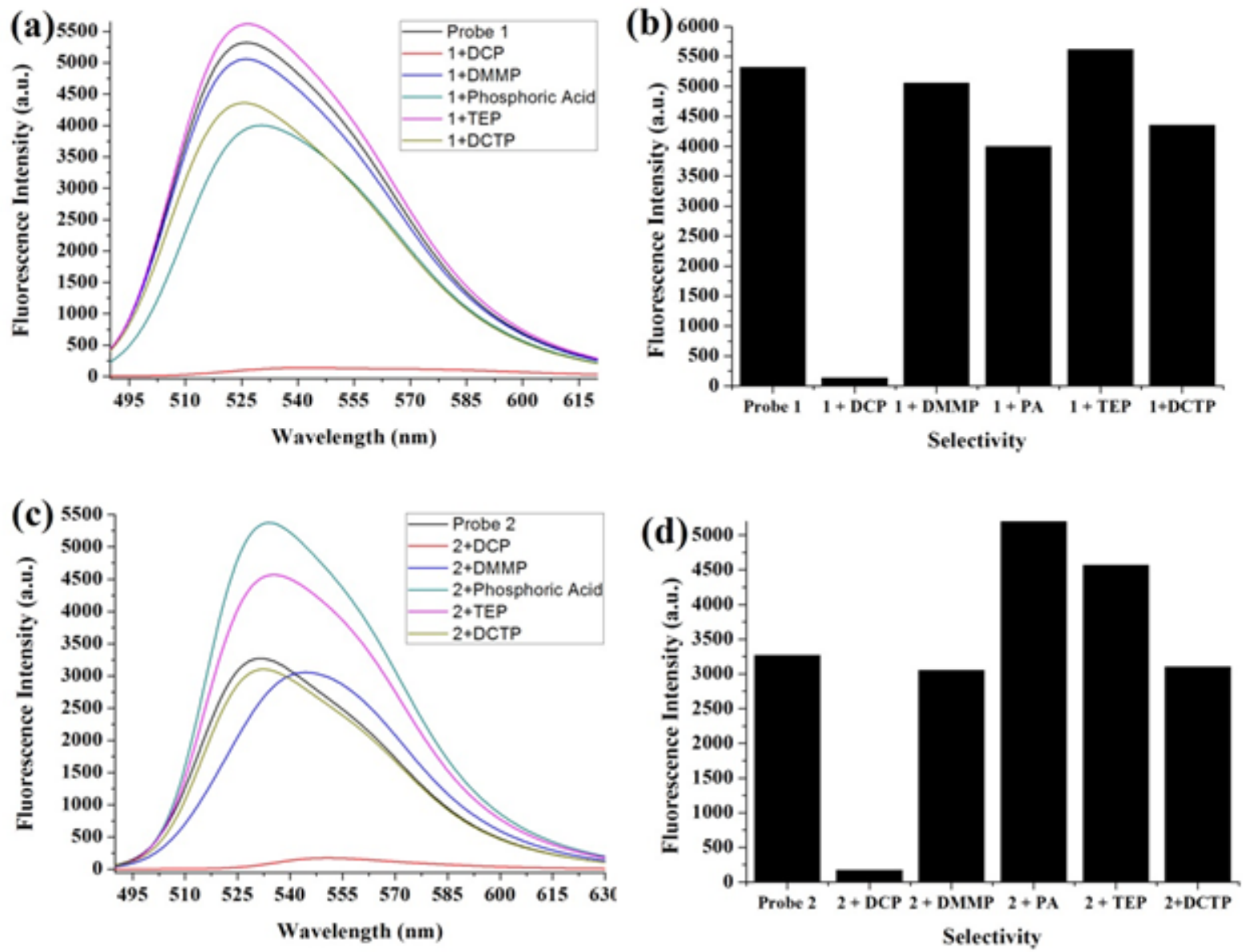

Fig. 6. Fluorescence selectivity comparison of (a,b) probe $\mathbf{1}(10.0 \mu \mathrm{M})$ in DMF (c,d) probe $2(10.0 \mu \mathrm{M})$ in dichloromethane with DCP, DMMP, phosphoric acid, triethyl phosphate and diethyl chlorothiophosphate. 


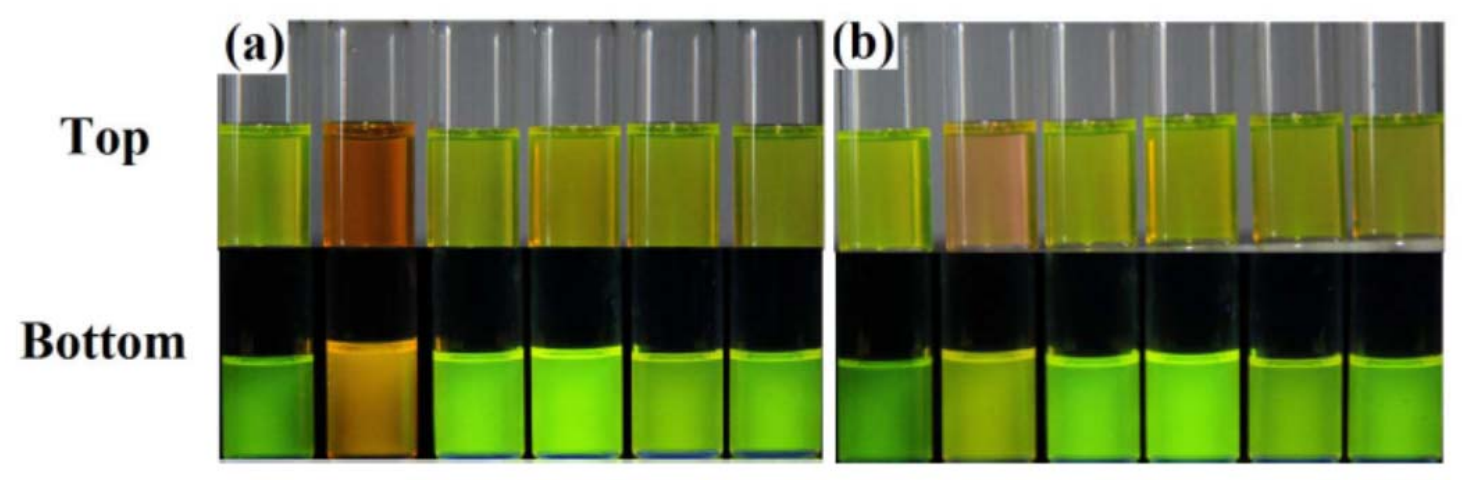

Fig. 7. Images of probes in the presense of other OPs. (a) is probe $\mathbf{1}(10.0 \mu \mathrm{M})$, while (b) is probe $2(10.0$ $\mu \mathrm{M})$ under visible (top) and UV light (bottom) with other OPs $(10.0 \mu \mathrm{M})$ from left to right: probes alone, probe + DCP, probe + DMMP, probe + phosphoric acid, probe + triethyl phosphate and probe + diethyl chlorothiophosphate. 

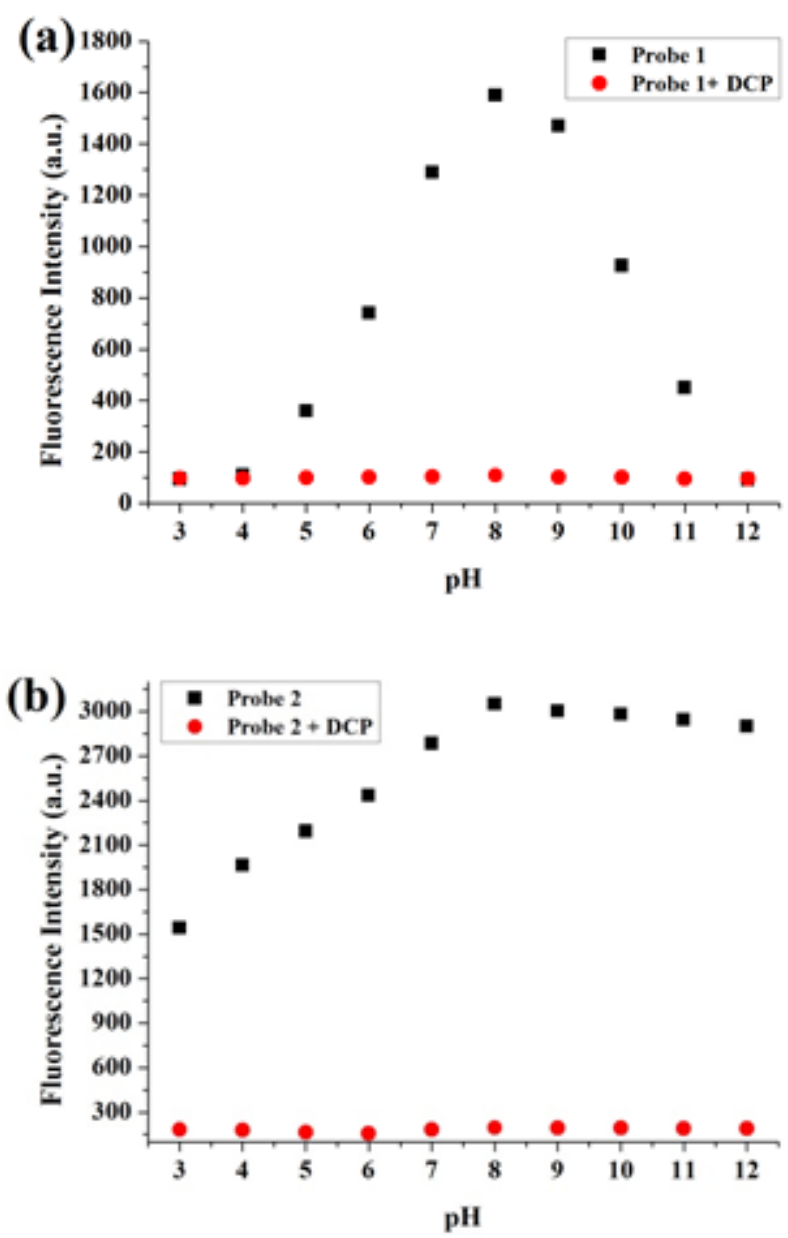

Fig. 8. The fluorescence intensity of a) probe $1(10.0 \mu \mathrm{M})$ at $526 \mathrm{~nm}$ in the presence and absence of DCP $(10.0 \mu \mathrm{M})$ in DMF, HEPES buffer $1: 1 \mathrm{v} / \mathrm{v}(10.0 \mu \mathrm{M})$ under different $\mathrm{pH}(3.0-12.0)$ and b) $2(10.0 \mu \mathrm{M})$ at $531 \mathrm{~nm}$ in the presence and absence of DCP $(10.0 \mu \mathrm{M})$ in DMSO, HEPES buffer $1: 1 \mathrm{v} / \mathrm{v}(10.0 \mu \mathrm{M})$ under different $\mathrm{pH}(3.0-12.0)$. 

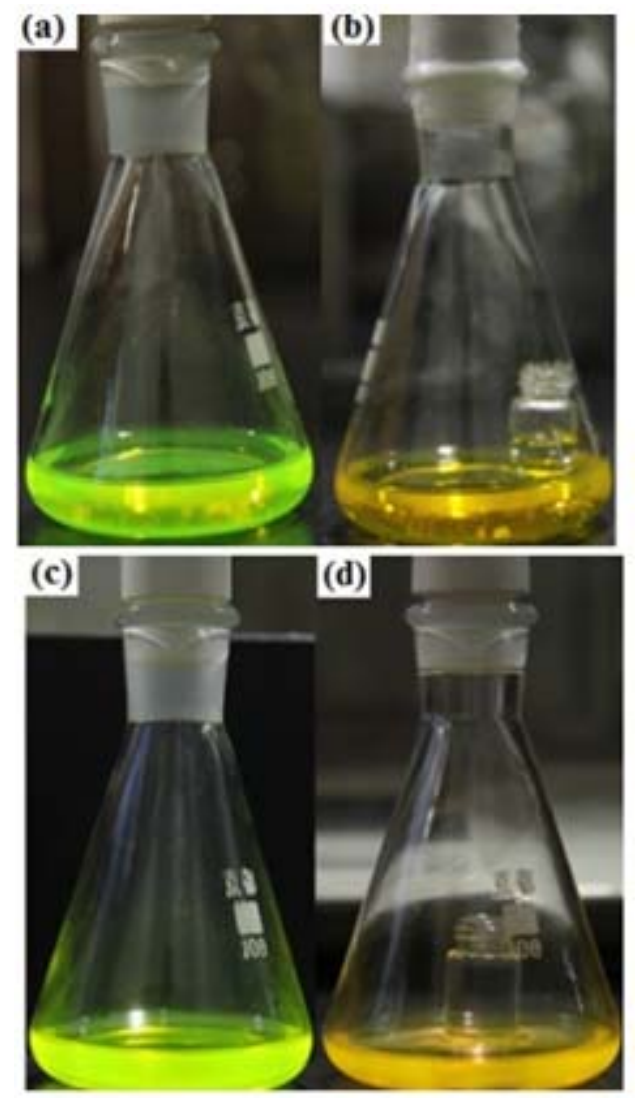

Fig. 9. Images of (a,c) free probe 1 and $2(10.0 \mu \mathrm{M})$, respectively, and (b,d) after exposure to DCP (15.0 $\mu \mathrm{M})$ vapors, respectively. 

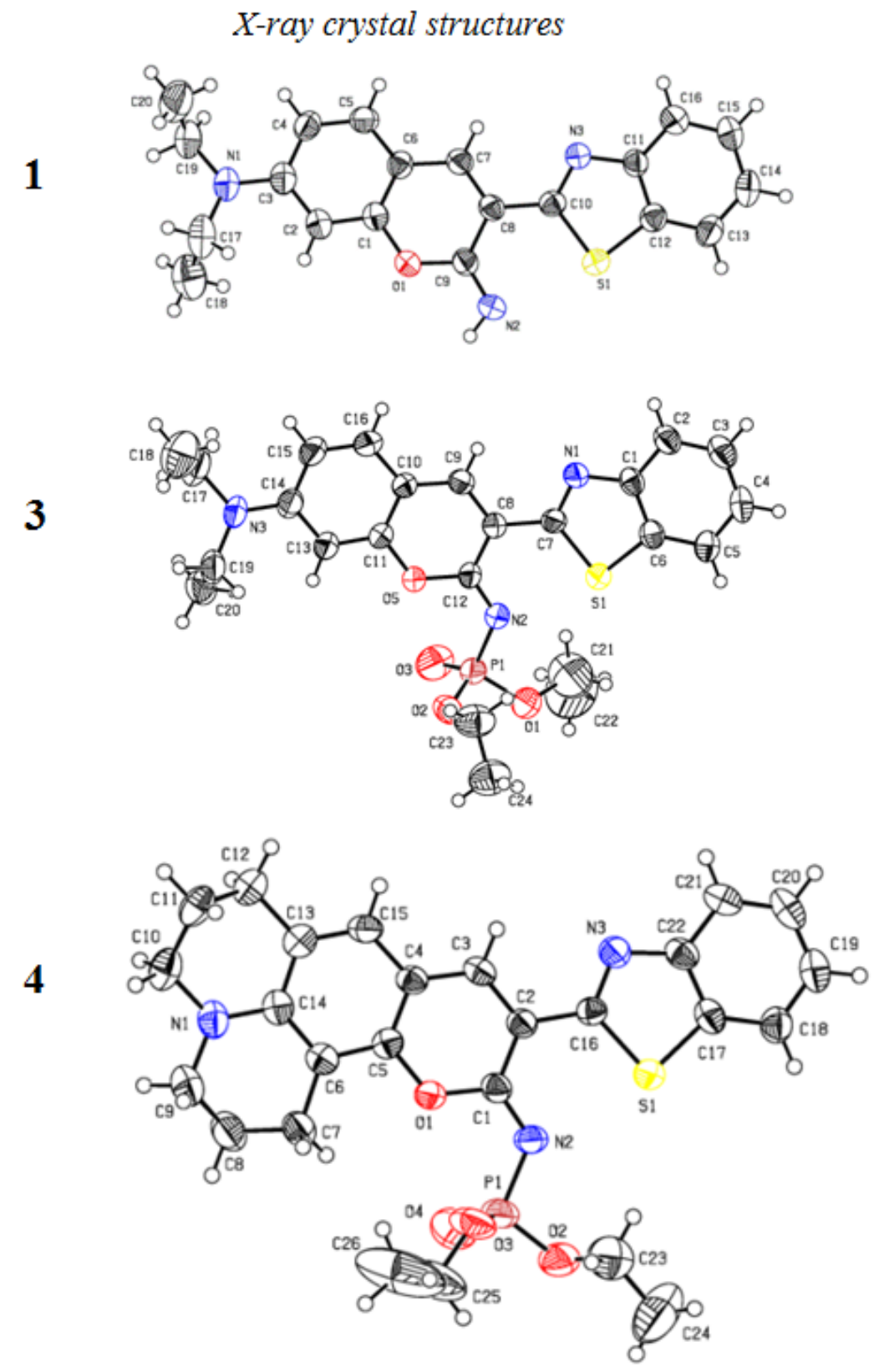

Fig. 10. View of the molecular structure of 1, 3 and 4 in the crystal. Thermal ellipsoids give $50 \%$ probability. 


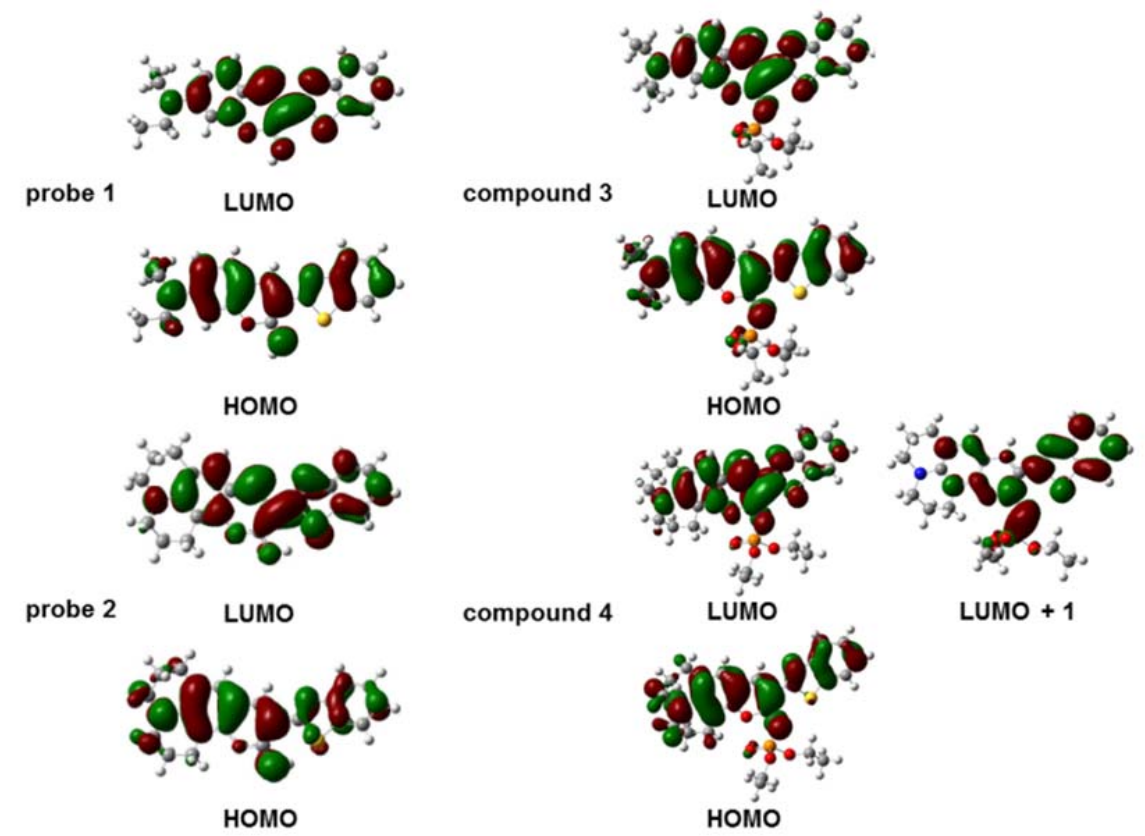

Fig. 11. HOMO-LUMO orbital plots of 1, 2, 3 and 4. 